\title{
Dynamic coronary MR angiography in a pig model with hyperpolarized water
}

\author{
Lipsø, Hans Kasper Wigh; Hansen, Esben Søvsø Szocska; Tougaard, Rasmus Stilling; Laustsen, \\ Christoffer; Ardenkjær-Larsen, Jan Henrik
}

Published in:

Magnetic Resonance in Medicine

Link to article, DOI:

$10.1002 / \mathrm{mrm} .27088$

Publication date:

2018

Document Version

Version created as part of publication process; publisher's layout; not normally made publicly available

Link back to DTU Orbit

Citation (APA):

Lipsø, H. K. W., Hansen, E. S. S., Tougaard, R. S., Laustsen, C., \& Ardenkjær-Larsen, J. H. (2018). Dynamic coronary MR angiography in a pig model with hyperpolarized water. Magnetic Resonance in Medicine, 80(3), 1165-1169. https://doi.org/10.1002/mrm.27088

\section{General rights}

Copyright and moral rights for the publications made accessible in the public portal are retained by the authors and/or other copyright owners and it is a condition of accessing publications that users recognise and abide by the legal requirements associated with these rights.

- Users may download and print one copy of any publication from the public portal for the purpose of private study or research.

- You may not further distribute the material or use it for any profit-making activity or commercial gain

- You may freely distribute the URL identifying the publication in the public portal 


\title{
Dynamic Coronary MR Angiography in a Pig Model With Hyperpolarized Water
}

\author{
Kasper Wigh Lipsø, ${ }^{1}$ Esben Søvsø Szocska Hansen, ${ }^{2,3}$ Rasmus Stilling Tougaard (ㄹ, ${ }^{2,4}$ \\ Christoffer Laustsen, ${ }^{2}$ and Jan Henrik Ardenkjær-Larsen ${ }^{1,5 *}$
}

Purpose: To investigate dynamic coronary MR angiography using hyperpolarized water as a positive contrast agent. Hyperpolarization can increase the signal by several orders of magnitude, and has recently been translated to human cardiac application. The aim was to achieve large ${ }^{1} \mathrm{H}$ signal enhancement to allow high-resolution imaging of the coronary arteries. Methods: Protons in $\mathrm{D}_{2} \mathrm{O}$ were hyperpolarized by dissolution dynamic nuclear polarization. A total of $18 \mathrm{~mL}$ of hyperpolarized water was injected into the coronary arteries of healthy pigs ( $N=9 ; 3$ injections in 3 animals). The MRI images were acquired with a gradient-echo sequence in an oblique slab covering the main left coronary arteries with $0.55 \mathrm{~mm}$ in-plane resolution. The acquisition time was $870 \mathrm{~ms}$ per frame.

Results: A more than 200-fold signal enhancement compared with thermally polarized water at $3 \mathrm{~T}$ was obtained. Coronary angiographic images with a signal-to-noise ratio from the left main stem of $269 \pm 169$ and coronary sharpness from the proximal left anterior descending coronary artery of $0.31 \pm$ $0.086 \mathrm{~mm}^{-1}$ were obtained. Dynamic images were acquired over a $10 \mathrm{~s}$ time window.

Conclusion: Hyperpolarized water MR angiography of the coronary arteries in a large animal model with high signal-to-noise ratio and high spatial and temporal resolution was obtained. Magn Reson Med 000:000-000, 2018. (c) 2018 International Society for Magnetic Resonance in Medicine.

Key words: coronary angiography; cardiac perfusion; magnetic resonance angiography; hyperpolarization; dissolutionDNP

\section{INTRODUCTION}

Magnetic resonance angiography (MRA) for coronary artery imaging has so far failed to translate into widespread clinical use $(1,2)$. This is partly because of the

\footnotetext{
${ }^{1}$ Department of Electrical Engineering, Technical University of Denmark, Kgs., Lyngby, Denmark.

${ }^{2}$ Department of Clinical Medicine, MR Research Centre, Aarhus University, Aarhus, Denmark.

${ }^{3}$ Danish Diabetes Academy, Odense, Denmark.

${ }^{4}$ Department of Cardiology Research, Aarhus University Hospital, Aarhus, Denmark.

${ }^{5}$ GE Healthcare, Brøndby, Denmark.

Grant sponsor: Independent Research Fund Denmark (12-127232) and Danish National Research Foundation (DNRF124).

${ }^{*}$ Correspondence to: Jan Henrik Ardenkjær-Larsen, Ph.D., DTU Electrical Engineering, Ørsteds Plads, Building 349, Room 126, 2800 Kgs. Lyngby, Denmark. E-mail: jhar@elektro.dtu.dk

Received 29 July 2017; revised 5 December 2017; accepted 22 December 2017

DOI 10.1002/mrm.27088

Published online 00 Month 2018 in Wiley Online Library (wileyonlinelibrary. com).
}

(C) 2018 International Society for Magnetic Resonance in Medicine inherent low sensitivity of MR, extending the acquisition time to several minutes and multiple respiratory and cardiac cycles (3). Unavoidably, motion will cause degradation of image quality even with the best correction schemes. Coronary arteries are challenging structures to image with MRI, because of the small and tortuous vessel structures. Coronary MRA has made great progress in recent years, but is still inferior to coronary CT angiography $(4,5)$.

One way to dramatically increase the sensitivity of MRI is hyperpolarization, in which the nuclear spin polarization is enhanced by several orders of magnitude (6). The method has recently been translated to human use for characterization of cancer (7) and assessment of myocardial metabolism (8) with hyperpolarized $\left[1-{ }^{13} \mathrm{C}\right]$ pyruvate. Other hyperpolarized ${ }^{13} \mathrm{C}$-labeled molecules have been demonstrated for angiography and perfusion (9-13). However, MRI of ${ }^{13} \mathrm{C}$ suffers from requiring specialized scanner hardware and coils, and a lack of optimized pulse sequences. In contrast, imaging of hyperpolarized water allows for the use of standard proton MR hardware and pulse sequences. Furthermore, protons have the advantage of a four times higher magnetic moment compared with ${ }^{13} \mathrm{C}$, resulting in a larger magnetization and higher signal-to-noise ratio (SNR). The lower gyromagnetic ratio of ${ }^{13} \mathrm{C}$ also limits the spatial resolution that can be achieved for the given gradient strength of the scanner. Hyperpolarized water has already been demonstrated for MRA in a rat model (14), and for imaging of the renal arteries and kidney perfusion in a pig model (15). Potentially, this method simultaneously allows for both coronary artery imaging and perfusion assessment.

The objective of this study was to investigate dynamic coronary MRA imaging using hyperpolarized water as a positive contrast agent in a large animal model.

\section{METHODS}

\section{Sample and Medium Preparation}

The sample preparation followed the methods published in (16). A sample of $1 \mathrm{~mL} 30 \mathrm{mM}$ TEMPO (2,2,6,6-Tetramethylpiperidine 1-oxyl, 98\%) in $\mathrm{H}_{2} \mathrm{O} /$ glycerol $1: 1(\mathrm{w} /$ w) was prepared. Dissolution medium consisted of $\mathrm{D}_{2} \mathrm{O}$ with $1 \mathrm{mM}$ calcium disodium ethylenediaminetetraacetic acid and 9g/L NaCl (all from Sigma Aldrich, Brøndby, Denmark).

\section{Fluid Path Filling}

The fluid path consisted of a syringe, from which the heated dissolution medium was transferred in an inner 
concentric tube to the sample vial for dissolution of the sample. The dissolved sample was transferred out of the magnetic field in the outer concentric tube.

The sample was placed in the vial using a $150 \mathrm{~cm}$ filling tube (17) and frozen in liquid nitrogen. A total of $31.01 \mathrm{~g}$ dissolution medium $(28 \mathrm{~mL})$ was filled in the syringe and degassed by bubbling with helium gas for $10 \mathrm{~min}$. The fluid path was flushed with helium gas for $2 \mathrm{~min}$, to remove air and ensure clear passage.

\section{Polarization}

The sample vial was rapidly transferred from the liquid nitrogen bath to the $5 \mathrm{~T}$ magnet in the polarizer (SPINlab, GE Healthcare, Brøndby, Denmark). The sample was irradiated with microwaves at $139.923 \mathrm{GHz}(50 \mathrm{~mW})$ for $1 \mathrm{~h}$.

\section{Dissolution}

The syringe containing the dissolution medium was heated to $130^{\circ} \mathrm{C} 1 \mathrm{~h}$ before dissolution. The piston in the syringe was driven with 16 bar pressure. The dissolved sample was injected into a sealed separatory funnel (150 mL) containing $25 \mathrm{~mL}$ of heptane (>99\%, Sigma Aldrich). The heptane had been degassed by flushing with helium gas in the funnel for $10 \mathrm{~min}$. The funnel was shaken heavily during dissolution for a total of $10 \mathrm{~s}$ from dissolution start. The lower, aqueous phase was extracted into a syringe before transferring to the scanner room. The proton concentration in the dissolved sample was analyzed by NMR after the addition of $0.2 \mathrm{M}$ potassium formate (Sigma Aldrich) as reference.

\section{Animal Protocol}

All animal experiments were carried out in compliance with the guidelines for use and care of laboratory animals and were approved by the Danish Inspectorate of Animal Experiments. Three healthy $30 \mathrm{~kg}$ female Danish domestic pigs were pre-sedated with intramuscular injection of Stressnil ( $1 \mathrm{~mL} / \mathrm{kg}$ ) and Midazolam ( $1 \mathrm{~mL} / \mathrm{kg})$, and anesthesia was maintained by continuous intravenous infusion of Propofol ( $0.4 \mathrm{mg} / \mathrm{kg} / \mathrm{h})$ and Fentanyl $(8 \mathrm{ng} / \mathrm{kg} /$ h). The animals were intubated and mechanically ventilated with a $60 \% \mathrm{O}_{2}$-air mix.

A $6 \mathrm{~F}$ introducer sheath was positioned in the left common femoral artery by ultrasound guidance to allow access for a $6 \mathrm{~F}$, JR 4.0 angiography catheter to be placed in the left anterior descending (LAD) coronary artery via $\mathrm{x}$-ray guidance. The angiography catheter was repeatedly flushed with heparinized sterile water to avoid occlusion. A total of $18 \mathrm{~mL}$ hyperpolarized water was injected over $5 \mathrm{~s}$, initiated approximately $22 \mathrm{~s}$ after dissolution.

\section{Magnetic Resonance Imaging Acquisition}

Magnetic resonance imaging detection was performed on a 3T GE HDx with an eight-channel cardiac array receiver coil (GE Healthcare, Milwaukee, WI, USA). Axial and coronal images were acquired to cover the heart to allow for planning of the slab for hyperpolarized water imaging. The sequence was as follows: a 3dimensional $\mathrm{T}_{1}$-weighted sequence with a standard steady-state free precession sequence (echo time $=1.1$ $\mathrm{ms}$, repetition time $=2.7 \mathrm{~ms}$, flip angle $=35^{\circ}$, image matrix $256 \times 256$, field of view $=340 \times 340 \mathrm{~mm}^{2}$, inplane resolution of $1.3 \mathrm{~mm}$ and slice thickness of $3 \mathrm{~mm}$ ). Angiograms were acquired in a tilted plane covering the LAD using a gradient-echo sequence with flip angle $=5^{\circ}$, slice thickness of $40 \mathrm{~mm}$, repetition time $=3.4 \mathrm{~ms}$, echo time $=0.984 \mathrm{~ms}$, matrix size $256 \times 256$, field of view$=140 \times 140 \mathrm{~mm}^{2}$, and in-plane resolution of $1.3 \mathrm{~mm}$. The acquisition time was $870 \mathrm{~ms}$ and no acceleration was used.

Nine injections were performed, with three in each animal. Region of interest analysis of the images was performed in OsiriX (Pixmeo, Geneva, Switzerland). Apparent signal-to-noise ratio (aSNR), defined as the ratio between the mean signal intensity in the vessel and the standard deviation of a background region of interest, and coronary sharpness, defined as the mean of the inverse distances between the 20 and $80 \%$ point on both sides of the 1dimensional cross-section profile of a coronary segment in the proximal $\mathrm{LAD}$, were measured to quantify imaging performance (18). The aSNR was measured at the left main coronary artery where the catheter was positioned (signal at bolus peak/standard deviation prior to injection), and coronary sharpness was measured in the proximal LAD at bolus peak (reported as the mean and standard deviation between the individual injections $(N=8$, one data point was discarded because of incorrect matrix size)). Data are presented as mean \pm standard deviation.

\section{RESULTS}

A water proton polarization of $5.3 \pm 0.9 \%$ and a $\mathrm{T}_{1}$ of $24 \pm 1$ s $(N=4)$ was measured by injecting the sample into a phantom placed in the $3 \mathrm{~T}$ scanner next to a thermally polarized water reference sample (15). The time from dissolution to measurement was $22 \mathrm{~s}$ on average. The average ${ }^{1} \mathrm{H}$ concentration was $3.8 \mathrm{M}$ in $\mathrm{D}_{2} \mathrm{O}$, and the produced volume was approximately $18 \mathrm{~mL}$.

Three healthy, Danish, domestic pigs weighing approximately $30 \mathrm{~kg}$ were included in this study. Multiple successive injections of hyperpolarized water and subsequent acquisitions of coronary angiograms were performed in each pig. Figure 1 shows angiograms from the three different pigs. The presented images are from the time point $5 \mathrm{~s}$ after injection of the hyperpolarized sample. The left main coronary artery, the LAD, and the left circumflex coronary arteries are visualized, along with their branches. Additionally, perfusion of the hyperpolarized water into the myocardial tissue can be noted. The presented images are acquired $5 \mathrm{~s}$ after injection of hyperpolarized sample, and some leaking into the vascular bed is observed in between arteries. Coefficients of variation for aSNR in the three animals were 12, 48 and 25\%, respectively. The aSNR from the left main stem was $269 \pm 169$, and coronary sharpness from the proximal LAD was $0.31 \pm 0.086 \mathrm{~mm}^{-1}$.

A representative time series of angiograms from a single injection is presented in Figure 2. Frames 1 to 12 display the first observed image with hyperpolarized signal and the 11 subsequent time steps of $1 \mathrm{~s}$ each. The delineation of the LAD appeared briefly as a result of the fast perfusion of hyperpolarized water into the tissue. Furthermore, sharp reduction in $\mathrm{T}_{1}$ when the hyperpolarized water mixed 
FIG. 1. Coronary angiograms after hyperpolarized water injections in three different animals. Images $5 \mathrm{~s}$ after injection of the hyperpolarized water.

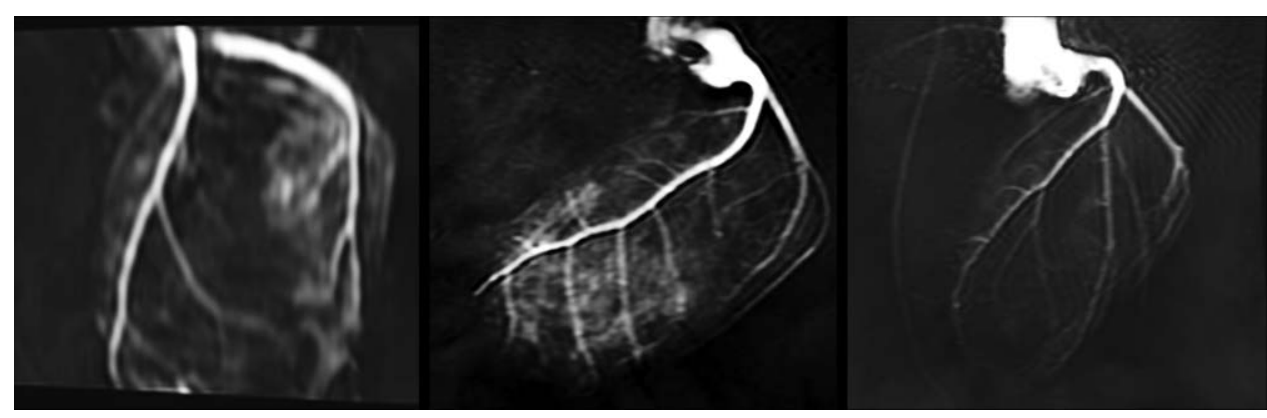

with blood probably contributed. No hyperpolarized signal was observed after $14 \mathrm{~s}$.

The signal in the minor branch arteries of the LAD appeared after the first few time steps, and grew increasingly more distinct throughout the series until vanishing. Similarly, the signal in the tissue occurred with a time delay from the arterial signal.

The temporal profile is shown in Figure 3. Average signal intensities in regions of interest positioned in the proximal LAD, medial LAD, and at a distal branch of LAD are plotted. Signal increase is first observed in the proximal LAD, and later in the medial and the distal branch of the LAD, as the bolus of hyperpolarized water passes through the coronary artery.

\section{DISCUSSION}

The main finding of this study was the demonstration of the feasibility of hyperpolarized water for dynamic coronary angiography. The coronary arteries were imaged with high spatial and temporal resolution as well as high SNR. The SNR found in this study is typically an order of magnitude higher than previous values reported for coronary MR angiography (19-21), for a higher in-plane spatial resolution. Admittedly, the acquisition scheme in this paper is a 2-dimensional projection as opposed to current state-of-the art of full 3-dimensional isotropic heart coverage. However, 3-dimensional acquisition would be possible with the relevant sequences available.

The images are acquired in less than $1 \mathrm{~s}$. Because of this relatively high temporal resolution, the perfusion of the myocardium may potentially be evaluated by this method. Increasing evidence support the altered myocardial perfusion as an accurate diagnostic marker of coronary artery disease (22). Assessment of perfusion using the presented method would require fitting of the signal with a model function, in which the relaxation of the signal is also taken into account. Contrary to most

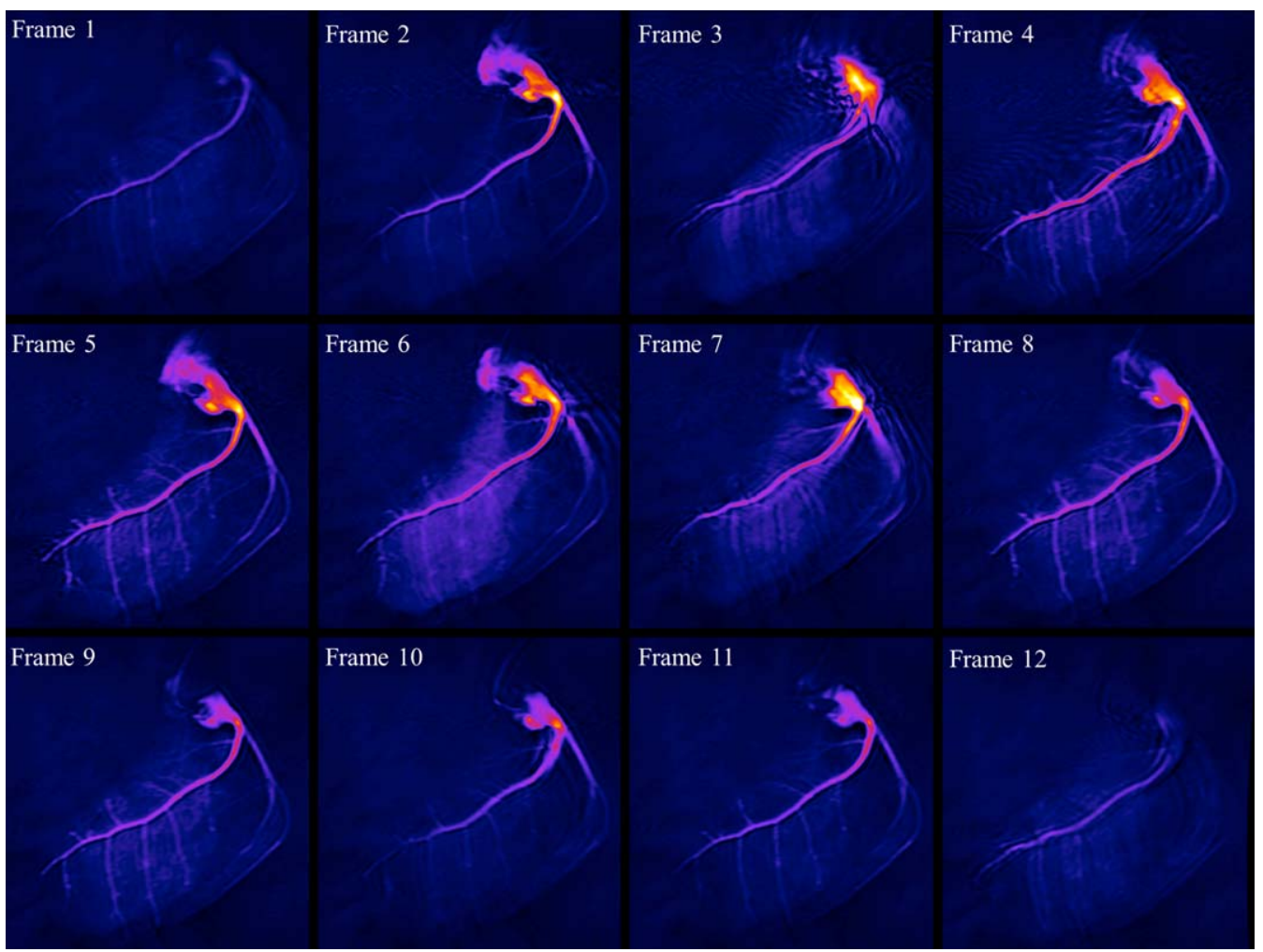

FIG. 2. Time series of consecutive images acquired every second from injection of sample. Major and minor vessels of the coronary tree can be easily identified. From animal displayed in the center of Figure 1. 


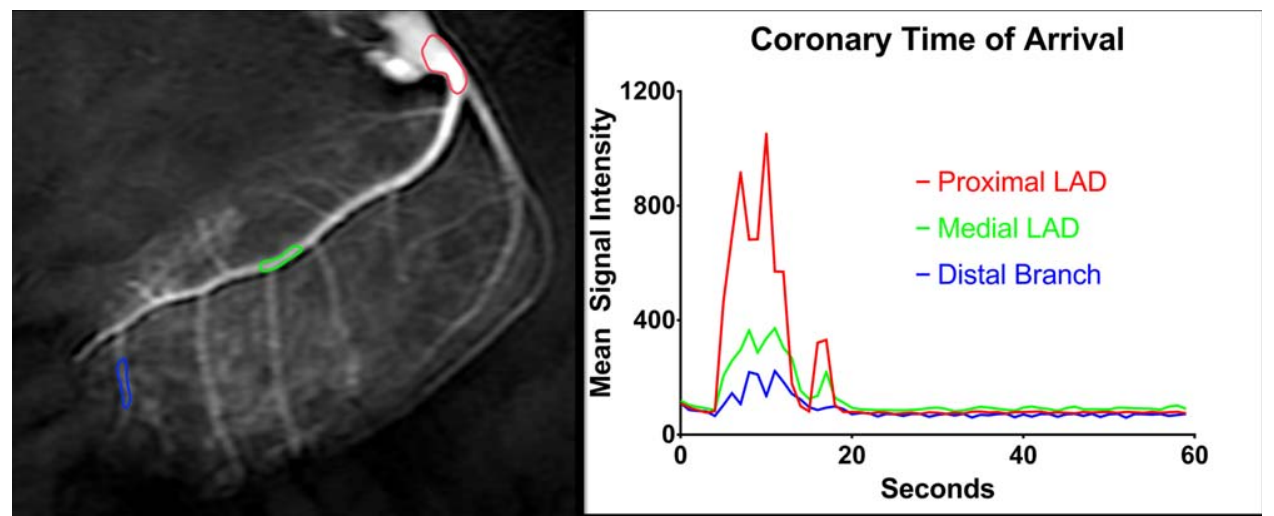

FIG. 3. Time of arrival of hyperpolarized water at proximal left anterior descending (LAD), medial LAD, and at a distal branch of LAD. From animal displayed in the center of Figure 1.

established perfusion measurement techniques, in which the perfusion of contrast-enhancing compounds, such as Gd-chelates, are used, myocardial perfusion measures for hyperpolarized water would yield positive contrast with the permeability characteristics of water.

The hyperpolarization method yielded consistent, reproducible sample properties (MR signal in particular). The images in this study were obtained with $5 \%$ proton polarization. We believe that 10 times higher polarization is achievable $(16,23)$, which would improve the image quality dramatically over what is presented here. The higher polarization could be obtained by reducing the relaxation loss in dissolution by more efficient removal of the radical, and by automated sample transfer.

\section{Limitations}

The main limitation for hyperpolarized water as an angiographic agent is the necessity for intra-arterial injection into the organ in question. This reason is the short relaxation time. After hyperpolarization, during handling, transfer and injection, the relaxation time is 20 to $30 \mathrm{~s}(15,24)$, but as soon as the water protons have been injected into the blood stream, the relaxation time shortens to a few seconds. Parawater has been a hot topic in MR in the past years, offering the prospect of extended relaxation times. So far, no one has succeeded, but a long-lived singlet state would allow for intravenous administration instead of catheterization, as well as more reliable perfusion and angiography. Another possible means of maintaining the magnetization for longer would be to encapsulate the hyperpolarized water in liposomes or similar, which would prevent the dilution with blood and the shortening of $\mathrm{T}_{1}$.

The motion of the heart is another key challenge for accurate measurements. The motion artifacts in this study may account for the signal fluctuations in the temporal profiles of Figure 3. Electrocardiogram-triggered acquisition and respiratory control (breath-hold) could reduce the motion artifacts observed in some frames, and will be required for perfusion analysis-something that was not available in the current setup. In general, the scanner used in this study was not optimized for cardiac imaging and the performance was inferior to current state-of-the-art (19-21). Likewise, for accurate bolus delivery and well-defined arterial input function, automated injection is required instead of the hand injection used in this study. Implementation of a power injector for bolus delivery is also a natural extension of the method to reduce time from dissolution to injection, thereby minimizing signal loss as a result of relaxation.

\section{CONCLUSIONS}

The consistently high quality of the images in a large animal model may have promise for future applications in humans for angiography and perfusion. The formulation was well tolerated by the animals; however, no formal safety studies have been performed to date. The dose of deuterium given was $0.5 \mathrm{~g} / \mathrm{kg}$ body weight, not far from the typical $0.2 \mathrm{~g} / \mathrm{kg}$ given with the water double labeling method $(25,26)$. The polarizer (SPINlab) has already been used for clinical studies with $\left[1-{ }^{13} \mathrm{C}\right]$ pyruvate (8), demonstrating the possibility of producing sterile solutions. The invasive nature of the arterial catheterization may be the largest challenge for clinical translation. However, future advances may allow for intravenous administration if the hyperpolarized water can be protected from relaxation (e.g., through an emulsion or encapsulation).

\section{REFERENCES}

1. Kato S, Kitagawa K, Ishida N, et al. Assessment of coronary artery disease using magnetic resonance coronary angiography. J Am Coll Cardiol 2010;56:983-991.

2. Kim W-Y, Danias PG, Stuber M, et al. Coronary magnetic resonance angiography for the detection of coronary stenoses. N Engl J Med 2011;345:1863-1869.

3. Kramer CM, Narula J. Atherosclerotic plaque imaging: the last frontier for cardiac magnetic resonance. JACC Cardiovasc Imaging 2009;2: 916-918.

4. Prompona M, Cyran C, Nikolaou K, Bauner K, Reiser M, Huber A. Contrast-enhanced whole-heart MR coronary angiography at $3.0 \mathrm{~T}$ using the intravascular contrast agent Gadofosveset. Invest Radiol 2009;44:369-374.

5. Bi X, Carr JC, Li D. Whole-heart coronary magnetic resonance angiography at 3 Tesla in 5 minutes with slow infusion of Gd-BOPTA, a high-relaxivity clinical contrast agent. Magn Reson Med 2007;58:1-7.

6. Ardenkjaer-Larsen JH, Fridlund B, Gram A, Hansson G, Hansson L, Lerche MH, Servin R, Thaning M, Golman K. Increase in signal-tonoise ratio of $>10,000$ times in liquid-state NMR. Proc Natl Acad Sci U S A 2003;100:10158-10163.

7. Nelson SJ, Kurhanewicz J, Vigneron DB, et al. Metabolic imaging of patients with prostate cancer using hyperpolarized [1- 13 C]pyruvate. Sci Transl Med 2013;5:198ra108. 
8. Cunningham CH, Lau JY, Chen AP, Geraghty BJ, Perks WJ, Roifman I, Wright GA, Connelly KA. Hyperpolarized 13C metabolic MRI of the human heart: initial experience. Circ Res 2016;119:1177-1182.

9. Golman K, Axelsson O, Jóhannesson H, Månsson S, Olofsson C, Petersson JS. Parahydrogen-induced polarization in imaging subsecond 13C angiography. Magn Reson Med 2001;46:1-5.

10. Golman K, Ardenkjær-Larsen JH, Svensson J, et al. 13C-angiography. Acad Radiol 2002;9:507-510.

11. Svensson J, Månsson S, Johansson E, Petersson JS, Olsson LE. Hyperpolarized 13C MR angiography using trueFISP. Magn Reson Med 2003;50:256-262.

12. Lipsø KW, Magnusson P, Ardenkjær-Larsen JH. Hyperpolarized 13C MR angiography. Curr Pharm Des 2016;22:90-95.

13. Olsson LE, Chai CM, Axelsson O, Karlsson M, Golman K, Petersson JS. MR coronary angiography in pigs with intraarterial injections of a hyperpolarized 13C substance. Magn Reson Med 2006;55:731-737.

14. Ardenkjaer-Larsen JH, Laustsen C, Bowen S, Rizi R. Hyperpolarized H2O MR angiography. Magn Reson Med 2014;71:50-56.

15. Lipsø KW, Szocska Hansen ES, Tougaard R, Laustsen C, ArdenkjærLarsen JH. Renal MR angiography and perfusion in the pig using hyperpolarized water. Magn Reson Med 2017;78:1131-1135.

16. Lipsø KW, Bowen S, Rybalko O, Ardenkjær-Larsen JH. Large dose hyperpolarized water with dissolution-DNP at high magnetic field. J Magn Reson 2017;274:65-72.

17. Malinowski RM, Lipsø KW, Lerche MH, Ardenkjær-Larsen JH. Dissolution dynamic nuclear polarization capability study with fluid path. J Magn Reson 2016;272:141-146.

18. Li D, Carr JC, Shea SM, Zheng J, Wielopolski PA, Finn JP. Coronary arteries: magnetization-prepared contrast-enhanced three-dimensional volume-targeted breath-hold MR angiography. Radiology 2001;219: 270-277.

19. Henningsson M, Hussain T, Vieira MS, Greil GF, Smink J, Ensbergen GV, Beck G, Botnar RM. Whole-heart coronary MR angiography using image-based navigation for the detection of coronary anomalies in adult patients with congenital heart disease. J Magn Reson Imaging 2016;43:947-955.

20. Addy NO, Ingle RR, Wu HH, Hu BS, Nishimura DG. High-resolution variable-density 3D cones coronary MRA. Magn Reson Med 2015;74: 614-621.

21. Nezafat M, Henningsson M, Ripley DP, Dedieu N, Greil G, Greenwood JP, Börnert P, Plein S, Botnar RM. Coronary MR angiography at 3T: fat suppression versus water-fat separation. MAGMA 2016;29:733-738.

22. Takx RAP, Blomberg BA, El Aidi H, Habets J, de Jong PA, Nagel E, Hoffmann U, Leiner T. Diagnostic accuracy of stress myocardial perfusion imaging compared to invasive coronary angiography with fractional flow reserve meta-analysis. Circ Cardiovasc Imaging 2015;8: e002666.

23. Harris T, Szekely O, Frydman L. On the potential of hyperpolarized water in biomolecular NMR studies. J Phys Chem B 2014;118:3281-3290.

24. Olsen G, Markhasin E, Szekely O, Bretschneider C, Frydman L. Optimizing water hyperpolarization and dissolution for sensitivityenhanced 2D biomolecular NMR. J Magn Reson 2016;264:49-58.

25. Kushner DJ, Baker A, Dunstall TG. Pharmacological uses and perspectives of heavy water and deuterated compounds. Can J Physiol Pharmacol 1999;77:79-88.

26. Speakman JR. Doubly labelled water: theory and practice. New York: Springer; 1997. 